\title{
AUTONOMIA PROFISSIONAL DO ENFERMEIRO: UMA CONOUISTA OBTIDA ATRAVÉS DE UM PROGRAMA DE EXTENSÃO À COMUNIDADE.
}

\author{
Mitsuko Ohnishi* \\ Arlete Bernine Fernandes e Silva** \\ Iwa K. Aida Utyama** \\ Nair Miyamoto Mussi*
}

\begin{abstract}
RESUMO - Os autores do presente trabalho, com o objetivo de enriquecer a formação dos alunos do Curso de Graduação em Enfermagem, propiciando experiências à saúde e ao trabalho em equipe multidisciplinar e, ainda, estender o campo de estágio aos alunos, na disciplina Fundamentos de Enfermagem, criaram um Programa de Extensão à Comunidade. A cada avaliação do Programa, vê os seus objetivos atingidos, tendo como destaque, a consolidação da autonomia profissional, tanto assistencial como administrativa.

ABSTRACT - The authoresses of the present work, on the purpose of enriching profissional education of undergraduate students of Nursing, providing them with learning experiences focused on primary health care and on working in multidisciplinaty team and, futhermore, to enlarge training opportunities to the students, developed in the discipline of Fundamental Nursing, an Extension Program to the Communit. Which has its objectives reached on each valuation of the project, with relevance on the consolidation of professional autonomy, related to assistantial and administrative aspects.
\end{abstract}

\section{INTRODUC̣ÃO}

O desenvolvimento industrial trouxe mudanças profundas na prática da assistência de enfermagem, a qual passou a ser predominantemente curativa. Com isto, as escolas de enfermagem adaptaram seus currículos para atender à demanda de preparar profissionais voltados para assistência de enfermagem em hospitais (SCHIMIT, 1984).

SOUZA (1981) coloca que o atual ensino de enfermagem proporciona aos estudantes a maior parte de suas experiências formativas nos cuidados com pacientes hospitalizados. Torna-se, então, incoerente e conflitante exigir-se-lhes, após formados, uma atuação compatível com as necessidades reais do país.

Acompanhando os modelos de currículo da maioria das escolas de enfermagem brasileiras, o ensino de enfermagem na Universidade Estadual de Londrina também estava voltado para área curativa, sendo que a assistência primária era muito limitada à teoria. Esta situação trazia inquietação e insatisfação por parte da disciplina de fundamentos de enfermagem.

COSTA (1978) refere que "ambulatórios são excelentes campos de estágio para estudantes de enfermagem, locais onde estes podem observar e executar práticas curativas e preventivas interrelacionadas". Porém, segundo OGUISSO (1984) poucas escolas of erecem campo de estágio em clínicas ou ambulatórios, de sorte que os alunos tenham condições de exercitar-se no campo de trabalho autônomo, experimentando de forma decisória o exercício liberal da profissão. Há ainda o agravante do enfermeiro exercer a profissão com independência e autonomia, criando creches, clínicas de repouso para idosos, ambulatórios de enfermagem, etc.

Com o objetivo de enriquecer a formação dos alunos através do trabalho em equipe multidisciplinar, estendendo o campo de estágio aos alunos do curso de graduação em enfermagem e com o intuito de propi-

\footnotetext{
* Professores Assistentes do Departamento de Enfermagem da Universidade Estadual de Londrina.

** Professores Auxiliares do Departamento de Enfermagem da Universidade Estadual de Londrina.
} 
ciar uma experiência de aprendizagem voltada à assistência primária à saúde e maior adequação entre o ensino teórico-prático, criou-se um programa de extensão à comunidade a nível ambulatorial em dezembro de 1981.

Este programa está sendo dinamizado no ambulatório do Hospital Universitário Regional do Norte do Paraná desde junho de 1982, contando com a participação de alunos e docentes do departamento de Enfermagem, Medicina e Serviço Social.

A função básica do docente de enfermagem neste programa vai ao encontro com a citação de COSTA (1978), onde atribui ao enfermeiro as atividades administrativas como planejamento, supervisão, organização, controle e avaliação das atividades desenvolvidas dentro do programa. Além desta, ainda é de sua função atividades de assistência, de ensino e de pesquisa.

Cabe ressaltar que, dentro das atividades desenvolvidas, é conferida ao docente e ao estudante de enfermagem total autonomia na sua área de atuação tanto administrativa como assistencial, de ensino e de pesquisa. Desta forma podemos dizer que, a área de enfermagem conseguiu atraveś deste serviço, ampliação de funções profissionais independentes.

A auto-determinação do enfermeiro dentro da equipe de saúde tem facilitado o trabalho da enfermeira, oferecendo a ela condições de prestar melhor assistência à saúde em quantidade e qualidade.

\section{O PROGRAMA}

\section{Objetivos:}

- Estender o campo de estágio aos alunos dos cursos de Enfermagem, Medicina e Serviço Social;

- Desenvolver no aluno uma visão do trabalho em equipe multidisciplinar;

- Contribuir para a formação profissional dos estagiários das áreas afins, mediante a sua atuação num relacionamento teórico-prático;

- Investigar nos clientes os problemas relacionados com fatores bio-psico-sociais e espirituais e capacitar o aluno a traçar um diagnóstico e elaborar um plano de atendimento;

- Orientar e encaminhar o cliente aos recursos da comunidade;

- Conscientizar o cliente de seus problemas e atuar como agente de mudança de comportamento, frente a sua problemática de saúde;

- Interpretar os fatores bio-psico-sociais e espirituais levantados pelas áreas afins, para melhor atuação da equipe de entendimento;

- Coletar subsídios para melhor adequação dos currículos às reais necessidades do ensino das áreas afins.

\section{IMPLANTAC̣ÃO E IMPLEMENTAC̣ÃO DO PROGRAMA}

\section{Caracterização da População}

Embora o programa tenha se iniciado em 1982, totalizando um atendimento de 7.588 clientes até junho de 1986, optou-se por descrever apenas os dados obtidos no período de janeiro a dezembro de 1985, para facilitar a compreensão e melhor situar o leitor no contexto do trabalho.

Os clientes são encaminhados pelos setores de internação e de triagem do Pronto Socorro, ambulatório e postos de saúde.

Os dados que apresentamos a seguir foram colhidos do livro registro e do prontuário do paciente.

Verificou-se uma alta percentagem de atendimento a clientes do sexo masculino, $69,1 \%$, sendo apenas $30,9 \%$ do sexo feminino; a maioria dos clientes, $91,3 \%$, reside em Londrina, sendo que a minoria, 1,3\%, pertence aos distritos.

Nota-se um maior atendimento dos clientes do FUNRURAL, 69,8\%, isto deve-se ao fato de ser o único hospital Regional credenciado para atender estes previdenciários. A maior porcentagem está na faixa de menos de 12 anos com $25,0 \%$, e em menor porcentagem está entre 53 a 62 anos com 7,6\%.

\section{Recursos Humanos}

O trabalho é desenvolvido por uma equipe composta pelos seguintes membros: quatro docentes de enfermagem, um de medicina, um docente do departamento de serviço social, um atendente de enfermagem e alunos dos cursos de graduação em enfermagem, medicina e serviço social.

A equipe é coordenada por um dos docentes de enfermagem e os demais atuam como supervisores de área, em escala de rodízio. Compete as docentes de enfermagem: o planejamento, coordenação, organização, controle, avaliação e promoção de ensino e pesquisa, bem como assistência direta ao cliente.

0 docente de medicina faz supervisão de área, participa de reuniões científicas e avaliação e reformulação do programa, presta assessoramento técnicocientífico à enfermagem, principalmente quanto à prescrição de drogas e fornecimento de atestados médicos.

Os alunos de medicina que estagiam na sala de curativo cursam o $5^{\circ}$. período, fazendo estágio extracurricular. Estes desenvolvem as seguintes atividades: organização da unidade, execução de técnica de curativo, registro das atividades assistenciais, orientação para auto-cuidado e encaminhamento a outros serviços.

$\mathrm{O}$ docente de serviço social orienta e supervisiona indiretamente os alunos do curso de serviço social, e os mesmos desenvolvem estágios curricular, recebem 
bolsa trabalho e fazem levantamento de problemas sociais.

Anualmente recruta-se uma média de 100 a 150 es- tagiários. A Tabela 1 é um demonstrativo da participação dos alunos no período de janeiro a dezembro de 1985.

TABELA 1 - Distribuição dos estagiários segundo o curso e o tipo de estágio.

\begin{tabular}{|c|c|c|c|c|c|c|c|c|}
\hline \multirow{2}{*}{$\begin{array}{l}\text { TIPO DE } \\
\text { ESTÁGIO } \\
\text { CURSO }\end{array}$} & \multicolumn{2}{|c|}{ CURRICULAR } & \multicolumn{2}{|c|}{ EXTRACURRICULAR } & \multicolumn{2}{|c|}{ BOLSISTA } & \multicolumn{2}{|c|}{ TOTAL } \\
\hline & $\mathrm{n}^{0}$ & $\%$ & $\mathrm{n} \stackrel{\mathrm{q}}{*}$ & $\%$ & $\mathrm{n}_{\stackrel{0}{0}}$ & $\%$ & $\mathrm{n}$ ? & $\%$ \\
\hline Enfermagem & 60 & 45,5 & 26 & 19,7 & 02 & 1,5 & 88 & 66,7 \\
\hline Medicina & - & - & 26 & 19,7 & 02 & 1,5 & 88 & 66,7 \\
\hline Serviço Social & - & - & - & - & 02 & 1,5 & 02 & 1,5 \\
\hline TOTAL & 60 & 45,5 & 68 & 51,5 & 04 & 3,0 & 132 & 100,0 \\
\hline
\end{tabular}

Destaca-se, nesta tabela, uma alta porcentagem de acadêmicos de enfermagem. Estão incluídos alunos do $4^{0}$. período em estágio curricular; de $5^{\circ}$ a $7^{\circ}$. período em estágio extracurricular e bolsistas. Os alunos do 4 . período desenvolvem atividades assistenciais, educativas e de organização diária do setor, com supervisão direta do docente de enfermagem. Os alunos de $5^{\circ}$. a $7^{\circ}$. períodos de enfermagem que desenvolvem estágio extracurricular, executam as seguintes atividades: estudo da dinâmica do programa, organização da unidade, avaliação diária da clientela presente, aplicação da metodologia de assistência através do processo de enfermagem, orientações para grupo de clientes previamente selecionados de acordo com as características das necessidades af etadas, encaminhamento de clientes para consultas às clínicas correspondentes, investigação das causas de abandono ao tratamento através de visitas domiciliares, participação em reuniões científicas e de avaliação do Programa e orientação aos alunos de medicina no que concerne à técnica de curativo, organização da unidade, registro das atividades assistenciais e educação individual para o auto-cuidado.

Ressaltamos que estes alunos têm inteira responsabilidade no andamento do serviço, quando da ausência do docente supervisor de área.

\section{Atuação Assistencial}

A consulta de enfermagem é realizada pelos docentes e alunos de enfermagem, sendo aplicada aos clientes previamente selecionados, de acordo com as seguintes características: clientes cujo tratamento depende de acompanhamento pelo problema específico da lesão ou daqueles que não têm condição de realizar o auto-cuidado, necessitando retornar ao ambulatório com freqüência.

Aos pacientes que apenas é feita retirada de pontos, não se aplica a metodologia na sua totalidade, orienta-se para o auto-cuidado e registra-se uma evolução completa.

As fases da consulta de enfermagem realizada na sala de curativo são as seguintes:

1. fase - Recepção do cliente.

2. fase - Histórico de Enfermagem.

3. fase - Diagnóstico e Plano Assistencial. Para a segunda e terceira fase é utilizado um impresso único para as três áreas atuantes (Anexo 1).

4 a fase - Evolução - contém todos os dados da implementação do plano assistencial, condições da lesão, permitindo um completo acompanhamento da evolução do cliente. Para o registro desta atividade é utilizado um impresso único para a área $\mathrm{e}$ de enfermagem.

5 . fase - Agendamento.

6 a fase - Encaminhamento ao serviço social, clínicas especializadas, posto de saúde/centro de saúde.

7. fase - Visita domiciliar.

Encontra-se na Tabela 2, as atividades assistenciais básicas de enfermagem, no período de janeiro a dezembro de 1985 . 
TABELA 2 - Distribuição das atividades assistenciais básicas de enfermagem no serviço, no período de janeiro a dezembro de 1985 .

\begin{tabular}{lrr}
\hline \hline & No & $\%$ \\
\hline Curativo & & \\
Orientação individualizada & 900 & 29,3 \\
Retirada de pontos & 803 & 26,1 \\
Encaminhamento & 158 & 5,1 \\
Palestra & 48 & 1,6 \\
Visita domiciliar & 30 & 1,0 \\
& & \\
TOTAL & $3075^{*}$ & 100,0 \\
\end{tabular}

* Alguns clientes receberam mais de uma atividade assistencial.

- Para a execução dos curativos é utilizada uma técnica elaborada pelas docentes de enfermagem a qual está registrada e à disposição dos estudantes.

- Orientação individualizada.

O incentivo ao auto-cuidado é observado rigorosamente. Sempre que o cliente tem condições para fazer o tratamento domiciliar, recebe todas orientações necessárias e o retorno é marcado apenas para avaliação e possíveis mudanças de conduta. Com is- to, reduz, consideravelmente, o número de atendimentos.

- Palestra.

As orientações para a atenção primária à saúde são realizadas através de palestras na sala de espera, tendo como ouvintes os clientes e acompanhantes específicos e também de outras clínicas. As palestras versam sobre os seguintes temas: primeiros socorros; higiene pessoal e ambiental; doenças transmissíveis; controle de insetos, roedores e artrópodes e saneamento básico.

- Encaminhamento.

Muitos clientes são encaminhados a postos de saúde próximos à sua moradia, pois não têem condições de realizar o tratamento domiciliar ou são de nível sócio-econômico carente, não podendo retornar com freqüência ao ambulatório para a avaliação. Para tanto é utilizado um impresso de referência/contra referência, com todas as informações necessárias.

- Visita domiciliar.

Quando são observados casos de abandono de tratamento, a investigação das causas é feita através de visita domiciliar, onde são detectados problemas sócio-econômicos, como por exemplo: falta de dinheiro e falta de transporte principalmente.

Através da visita domiciliar o aluno tem oportunidade de conhecer a realidade do cliente, facilitando assim a adequação das orientações.

Existe um fluxograma a ser seguido pelo cliente para o seu atendimento no serviço (Figura 1).

FIGURA 1 - Fluxograma para atendimento do cliente na sala de curativo do hospital universitário regional do norte do Paraná.

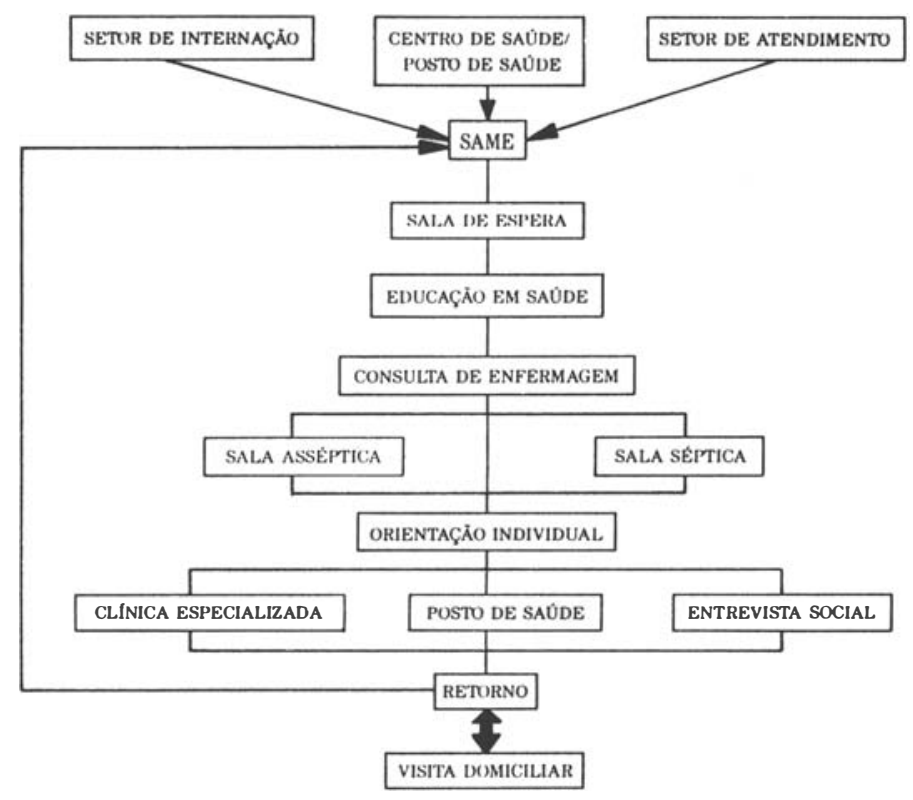




\section{Atuação administrativa}

0 pessoal de enfermagem desenvolve as seguintes atividades administrativas:

- Planejamento da área física com participação ativa nos estudos de reforma para adaptação às necessidades do programa;

- Elaboração do manual de rotinas administrativas, de funcionamento e de atendimento.

- Divulgação do programa aos Departamentos do Hospital Universitário Regional do Norte do Paraná, aos órgãos competentes e as comunidades envolvidas, através dos órgãos de comunicação de massa.

- Seleção dos estagiários inscritos.

- Elaboração de escalas de atuação de acordo com os critérios estabelecidos pelos cursos atuantes no programa.

- Distribuição das tarefas diárias.

- Reuniões científicas.

- Avaliação periódica do programa.

- Avaliação geral, relatório e reformulação do Programa.

\section{COMENTÁRIOS}

0 programa desenvolvido por uma equipe multidisciplinar traz benefícios para estagiários, clientes e para o próprio serviço, fazendo-nos acreditar que esta atividade é indispensável ao processo ensino aprendizagem.

Acreditamos que quando os alunos de enfermgem vivenciam o trabalho autônomo do enfermeiro, repercute na formação dos mesmos, assumindo responsabilidades clínicas nos serviços de saúde.

Observa-se que o nível de valorização é muito grande pela execução autônoma das atividades tanto por parte do docente quanto do aluno de enfermagem, por permitir autonomia na tomada de conduta terapêuti- ca adequada, facilitando o acompanhamento da evolução clínica do cliente. Esta colocação vai ao encontro com as idéias de WAGNER citado por SCHIMIDT (1978), afirmando que "o enfermeiro é o elemento da equipe de saúde mais apropriado a assumir a coordenação do planejamento dos cuidados do paciente, conjugando as diversas prescrições no plano integrado e assistencial".

\section{CONCLUSÕES E RECOMENDAC̣ÕES}

Observamos um alto nível de satisfação profissional do pessoal de enfermagem, envolvido no programa.

Acreditamos que com esta dinãmica de trabalho estamos contribuindo em parte para a formação de enfermeiros generalistas, vivenciando experiências que não seriam encontradas somente à nível de assistência a clientes em regime de internação.

Recomendamos que as escolas de enfermagem proporcionem estágios aos seus alunos, procurando integrar a assistência primária, secundária e terciária no ensino, a nível ambulatorial, para prestação de serviços à comunidade e ainda utilizar creches, escolas, asilos e outros criando novos campos de trabalho para o enfermeiro. Recomendamos, também, a continuidade desta luta na conquista de novos campos de trabalho.

\section{REFERÊNCIAS BIBLIOGRÁFICAS}

1. COSTA, M.I.C. Atuação do enfermeiro na equipe multiprofissional. Revista Brasileira de Enfermagem, Distrito Federal, 31:321-39, 1978.

2. OGUISSO, T. Ampliação de funções do enfermeiro. Revista Paulista de Enfermagem, São Paulo, 4(3): 95-8, jul./set. 1984.

3. SCHIMIDT, M.J. Natureza das condições de trabalho da enfermagem. Revista Paulista de Enfermagem, São Paulo, 4(3): 89-94, jul./set. 1984.

4. SOUZA, A.M.G. et alii. Saúde e Ensino de Enfermagem no Brasil - Condições sobre o parecer 163/72. Revista Brasileira de Enfermagem, Distrito Federal, 34:182-88, 1981. 


\section{ANEXO I}

\section{HOSPITAL UNIVERSITÁRIO REGIONAL DO NORTE DO PARANÁ}

SUBPROJETO: “ASSISTÊNCIA PRIMÁRIA À SAÚDE AO PACIENTE PORTADOR DE FERIDA: ATUAÇÃO DE UMA EQUIPE MULTIDISCIPLINAR"

\section{I - IDENTIFICAÇÃO}

Nome: Registro:

Data de nascimento: L_ Sexo: Cor: Categoria:

Estado Civil: Profissão:

Escolaridade: Natural:

Endereço residencial:

Data de atendimento:

II - COMPOSIÇÃO FAMILIAR

Nome Parente Idade Instrução Profissão Salário

III - SITUAÇÃO HABITACIONAL

Casa: Própria: Cedida: Alugada:

Alvenaria: Madeira: Outros:

Números de cômodos: № de leitos:

Banheiro: Sim Tipo:

Não $\square$

IV - DIAGNÓSTICO E PLANO ASSISTENCIAL

A - Área de Enfermagem:

Assinatura:

COREn:

Data:

116 - Rev. Bras. de Enf., Brasília, 40 (2/3) abri/maio/jun./jul./ago./set., 1987 
B - Área de Medicina:

Assinatura:

Data:

C - Área de Serviço Social:

Assinatura:

Dat a :

OBSERVAÇÕES:

Assinatura;

COREn:

Data: 